\title{
UNA NUEVA DEDICACIÓN A MARTE EN BEMBIBRE (LEÓN) ${ }^{1}$
}

\section{A NEW DEDICATION TO MARS AT BEMBIBRE (LEÓN)}

\author{
M. a Cruz González Rodríguez y Manuel Ramírez Sánchez \\ UPVIEHU - UNIVERSIDAD DE LAS PALMAS DE GRAN CANARIA \\ cruz.gonzalez@ehu.eus \\ manuel.ramirez@ulpgc.es
}

DOI: $10.1387 /$ veleia.14995

\begin{abstract}
Resumen: Se da a conocer una inscripción inédita procedente de San Román de Bembibre (León), que se conserva reutilizada en la Iglesia de San Román Mártir. El texto es muy breve, pero incluye un epíteto inédito, Propitius, en las inscripciones hispánicas dedicadas a esta divinidad.

Palabras clave: Epigrafía romana, Mars Propitius, Conventus Asturum, Hispania citerior.

Abstract: We present an inedited inscription from San Roman de Bembibre (León) which is preserved in San Roman Mártir Church reused as building material. The text is very short but it includes the epithet Propitius, which is new among the Hispanic inscriptions dedicated to this deity.

Keywords: Roman Epigraphy, Mars Propitius, Conventus Asturum, Hispania citerior.
\end{abstract}

Recibido: 12-06-2015

Definitivo: 13-06-2015

\section{DesCRIPCIÓN Y EDICIÓN DE LA PIEZA}

La inscripción que aquí presentamos se descubrió en el año 2008, en el transcurso de unas obras llevadas a cabo en la iglesia de San Román Mártir, en la localidad de San Román, pedanía de Bembibre (León), al quitar el encalado de las paredes para dejar la piedra vista. El epígrafe se conserva reutilizado como sillar en un pilar del crucero situado en el lado de la Epístola, donde la estudiamos el 14 de abril de $2015^{2}$. El material en el que está realizada es el conocido como granito de Monte Arenas (Ponferrada, León) y se encuentra dispuesta en posición horizontal, en la cuarta hilada de la pilastra (Figura 1). Debido a su reaprovechamiento como material de construcción,

${ }^{1}$ Este trabajo se enmarca en el Proyecto de Investigación HAR2011-25370 financiado por el Ministerio de Ciencia e Innovación, Dirección General de Investigación y Gestión del Plan Nacional de I + D + i.
2 Agradecemos a Manuel Olano Pastor la información sobre la existencia de esta inscripción, la fecha y circunstancias de su descubrimiento, así como su amabilidad y disponibilidad para acompañarnos durante la autopsia de la pieza. 


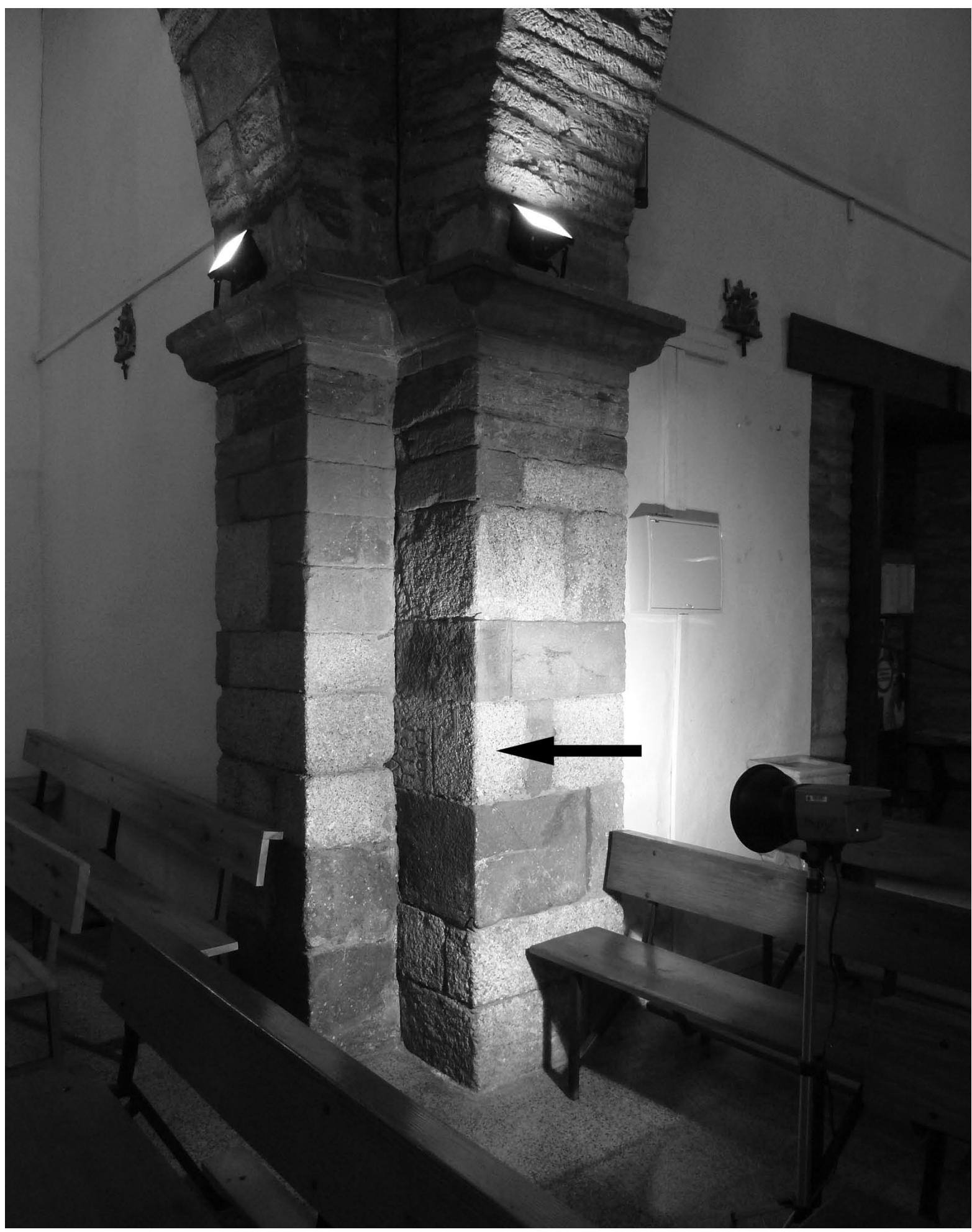

Figura I. Localización del epígrafe en la Iglesia de San Román Mártir (Bembibre, León). Fotografía de los autores 
el soporte epigráfico original, que debió ser un ara, fue alterado para encajarlo en la construcción como sillar. La base del ara, a pesar de haber sido rebajada, aún conserva la línea de la moldura que delimitaba el campo epigráfico en su parte inferior y, a pesar del rebaje realizado en la base, esta sobresale levemente con respecto al fuste original de la pieza.

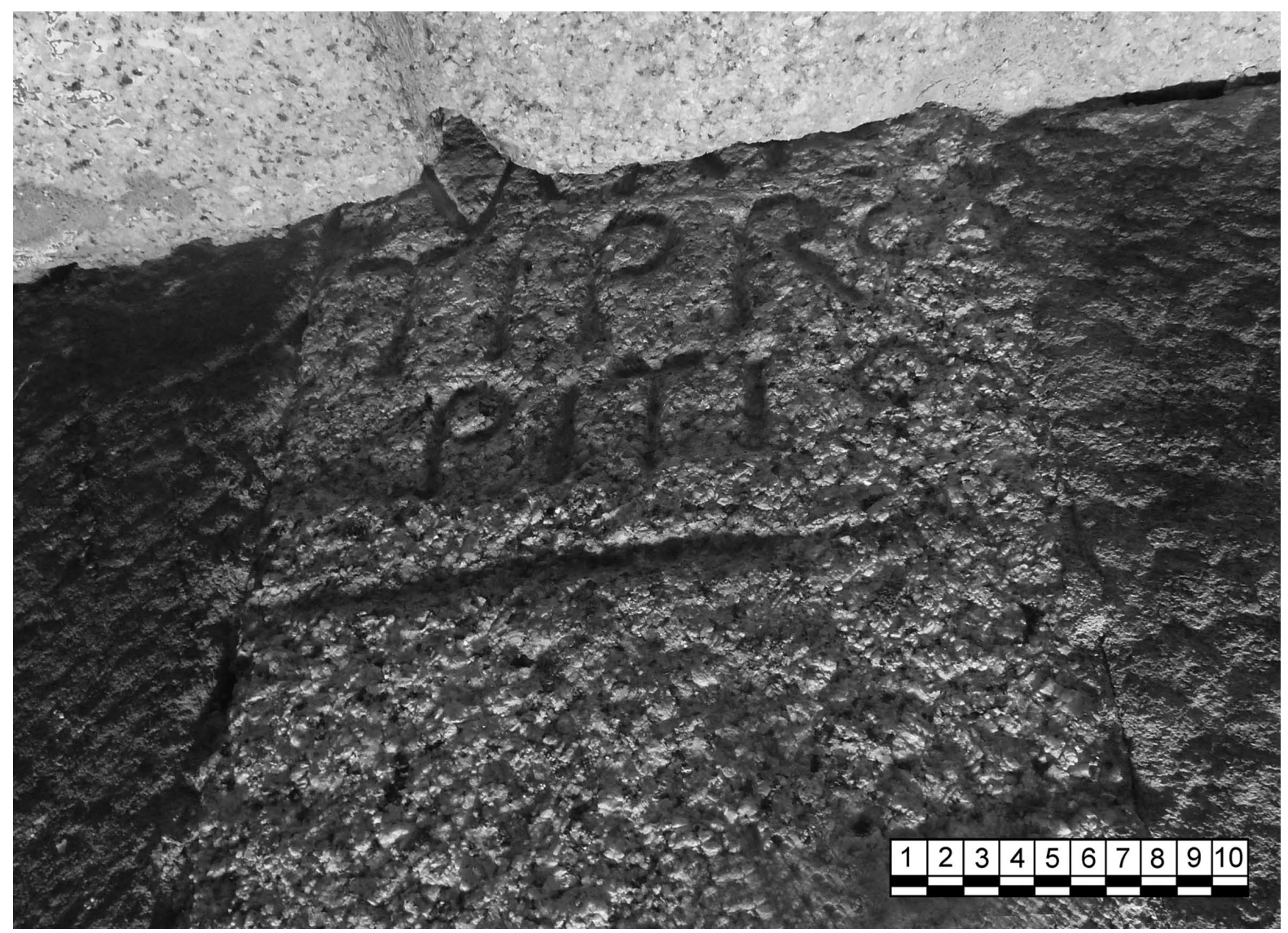

Figura 2. Inscripción dedicada a Mars Propitius. Fotografía de los autores

Las dimensiones de la inscripción son [42] $\mathrm{cm} \times 31 \mathrm{~cm} \times 21 \mathrm{~cm}$. La posición de la pieza, que se introduce en la crujía del pilar, sólo permite leer las dos últimas líneas del texto y la parte inferior de las letras escritas en la línea inmediatamente anterior, sin que sea posible determinar si la inscripción posee más líneas de texto, ocultas bajo la piedra. Las letras capitales, de factura regular y ejecutadas con sección en $\mathrm{V}$, miden $7 \mathrm{~cm}$ en la línea 2, excepto la $\mathrm{O}$, que mide $3 \mathrm{~cm}$; mientras que en la línea 3 miden $5 \mathrm{~cm}$, excepto la $\mathrm{O}$, que también mide $3 \mathrm{~cm}$. No es posible determinar la altura de las letras de la línea 1, pero su anchura (por ejemplo, la $\mathrm{M}$ mide $9 \mathrm{~cm}$ de ancho) posiblemente indica que también midan $7 \mathrm{~cm}$, como en la línea 2 . No se aprecian interpunciones y llama la atención el espacio vacío al comienzo de la l. 3, posiblemente para justificar el texto en el lado derecho del campo epigráfico. Es digno de destacar que la $\mathrm{M}$ de la 1.1 es muy abierta y la A carece de travesaño, mientras que la $\mathrm{R}$ de la 1.2 , también muy abierta, no llega a unir la panza y el tercer trazo con el primero (Figura 2). 
El texto dice:

\section{Mar \\ ti Pro \\ pitio}

La inscripción carece de las fórmulas habituales en este tipo de dedicaciones y, debido a su emplazamiento — tal y como ya hemos señalado — no es posible determinar si hay más líneas de texto encima de las tres que se pueden ver. En caso de que se conserven más líneas de texto, cabe pensar que en ellas aparecería mencionado el nombre del dedicante, si es que este llegó a grabarse.

\section{Apuntes sobre las dedicaciones a Marte en la zona y el nuevo epíteto}

Este nuevo testimonio epigráfico viene a sumarse a los cuatro epígrafes hasta ahora conocidos y dedicados a esta divinidad en esta provincia: Mars Tillenus en Quintana del Marco (ERPLe 22); Mars Gradivus y Mars Sagatus en Astorga (Mañanes 1982, n. ${ }^{\circ} 14$ y 16) y, el hallazgo más reciente dedicado a Mars Cemenilus en la ciudad de León (González Rodríguez 2013, 15-17) 3 .

Lo más novedoso de la inscripción que aquí presentamos es la utilización del adjetivo propitius como epíteto del nombre de la divinidad lo que convierte esta pieza en el primer testimonio de tal determinante en la epigrafía hispánica. Sin embargo, no es la primera vez que este vocablo se documenta en fórmulas votivas de inscripciones del Occidente peninsular. Hasta el momento se conocen tres testimonios epigráficos, uno de Condeixa-a-Velha, Condeixa-a-Nova (Coimbra, Portugal), en una inscripción que, muy posiblemente, está dedicada también al dios Marte ${ }^{4}$; en la conocida como tarifa sacrificial de Penafiel (Porto) ${ }^{5}$ y en un epígrafe de San Miguel de Mota, Terena (Alandroal, Évora) dedicado a Endovelico ${ }^{6}$.

Esta misma expresión se registra, igualmente, en las fuentes literarias asociada también a Marte, tal y como se comprueba en la invocación a Mars Pater que podemos leer en el Tratado de Agricultura de M. Porcio Catón, en la plegaria realizada en el contexto del ritual de purificación de un campo: "Mars pater, te precor quaesoque uti sies volens propitius mibi domo familiaeque nostrae... (Catón, Agric., 141, 2) y cuya finalidad es invocar la protección y el favor de la divinidad sobre la propiedad agrícola y la familia ${ }^{7}$, sobre la salud de las personas y la fertilidad de la tierra. Si el dios

3 En el conjunto de la Hispania citerior se contabilizan en torno a una veintena de dedicaciones a Marte. Cf. Le Roux 2006, 88. Para los hallazgos del Noroeste vid. Tranoy 1981, 314-315 y González Rodríguez 2013.

4 Etia[m] quotsi r[ogatu meo] atnueris [propitius] aram dic[abo cum hostiis] auratis et [---] taurum $m$ [actabo in] ariis Ma[rtis Aug(usti)] (Alarcao, Étienne 1976, n. $\left.{ }^{\circ} 19=A E 1975,480\right)$. En este caso el adjetivo se refiere al devoto y no, como en el que nos ocupa, a la divinidad.

5 votum solvit propitius sis rogo (CIL II 6259, $24=$ AE 1999, 897).
6 ...merito hun $[c]$ deum sibi propitium (IRCP 495).

7 «Padre Marte, ruégote y suplícote seas benévolo y propicio a mi, a mi casa y a nuestros esclavos...en virtud de ello he ordenado que los suovetaurilia circunden mi campo, mi tierra y mi propiedad para que tú impidas, apartes y desvíes las enfermedades visibles e invisibles, la infecundidad, las calamidades y las inclemencias...". Traducción tomada de A. García-Toraño, Catón el Censor. Tratado de Agricultura. Fragmentos, Madrid 2012, Ed. Gredos. El mismo adjetivo se repite en la misma obra (Agric., 134, 2) en sendas plegarias, similares a la vista para Marte, y dirigidas, en este caso, a Jano y a Júpiter, respectivamente. 
es propicio (favorable, benigno) la cosecha será exitosa y la salud de la familia buena. A cambio, como es norma en la religión romana, el propietario ofrece un sacrificio a Marte, una suovetaurilia lactentia ${ }^{8}$.

Estos ejemplos muestran que, trátese de un uso epigráfico o literario, el adjetivo propitius,-a,um (propicio, favorable, benigno, aplacado, indulgente") es un vocablo "de la langue religieuse qui s'applique aux dieux» (Ernout, Millet 1994 [1932], 539) y en el marco de la religio el mecanismo que el devoto posee para lograr que la divinidad se torne propicia no es otro que el rito conforme a las reglas del ius sacrum. Mediante la imprecación pronunciada adecuadamente y la realización de un sacrificio, sea de ofrendas animales o vegetales, en función del tipo de divinidad, el hombre busca conseguir el favor de la divinidad invocada.

En el caso que nos ocupa este adjetivo se ha convertido en epíteto del nombre de la divinidad otorgándole una definición y función precisas: Marte Propicio (Marte Benévolo), es decir, el Marte Indulgente que concede lo que el devoto le pide. Ya no se trata, como con la plegaria, de «convencer» a la divinidad de que sea favorable sino que esta ya ha adquirido este calificativo y, en consecuencia, actúa como tal dios benévolo y complaciente respondiendo positivamente a las invocaciones, peticiones y ruegos de los cultores.

La utilización de propitius como epíteto no es abundante en la epigrafía del Imperio. La búsqueda en la Epigraphik-Datenbank Clauss / Slaby (EDCS) devuelve 22 correspondencias del mismo adjetivo en dativo, frente a 13 testimonios en nominativo, aunque los ejemplos en los que este aparece referido a una divinidad en concreto son relativamente escasos: dos ejemplos referidos a Mercurio (CIL XII, 2440; RIB 1, 244), uno a Apolo (CIL III, 7463), uno a Dolichenus (AE 2004, 1387), uno más referido a los Lares (CIL IV, 844), y otro a los Penates (CIL XI, 1286). También hay varios testimonios referidos a divinidades locales, como el dios galo Brixantos en la provincia Lugudunense (CIL XIII, 2812) o el dios Cilvanos en la provincia de Panonia inferior (TitAq 1,300$)^{10}$.

A estos escasos testimonios se suma ahora el epígrafe de Bembibre que, a su vez, completa la lista de los epítetos clásicos (más abundantes que los locales) conocidos hasta el momento en la epigrafía hispana: Ultor, Campester, Augustus, Pater, Gradivus, Sagatus ${ }^{11}$.

Lamentablemente, la reutilización edilicia (Di Stefano Manzella 1987, 71) de la inscripción de Bembibre, al margen de la falta de contextualización y la consiguiente falta de información sobre su procedencia, nos impide saber si el dedicante aparece mencionado en las hipotéticas primeras líneas del texto. Esta característica, unida a la ausencia de fórmulas finales - como suele ser frecuente en la epigrafía votiva de la zona - que nos impide saber, de forma totalmente segura, si estamos ante una inscripción votiva, son un obstáculo insalvable para poder hacer alguna valoración

Igualmente, a Marte, en un contexto ritual en el que colabora con la diosa agraria Dia, se implora también (por tres veces) en un carmen arval, en el marco de un rito in luco por medio del cual los fratres arvales piden su protección, en su calidad de "guardian», sobre el territorio de Roma. Vid. Scheid 1990, 621-623.

${ }^{8}$ En el caso de Jano y Júpiter son ofrendas vegetales.

9 Diccionario Latino-Español. Valbuena reformado de M.D.P. Martínez López, Paris-México 1926, 701. Puede verse también: S. Segura, Nuevo diccionario etimológico Latin-Español y de las voces derivadas, Bilbao
2001, 609: "propicio [refer. espec. a los dioses], bien dispuesto, favorable, benévolo...»; Oxford Latin Dictionary. Fascicle V, Oxford 1976, 1493 y F. Gaffiot, Dictionnaire Latin Français, Paris 1934, 1258.

10 Consulta realizada con los términos de búsqueda "propitio" y "propitius» el 7 de junio de 2015. En acusativo se constatan también algunos ejemplos relacionados con Sucelo (3 testimonios) y Júpiter (1 testimonio) en la Galia Narbonense; Mercurio en Retia o Saturno en Numidia.

11 Sobre el significado y valoración de este último epíteto vid. Le Roux 2006, 89-90. 
sobre las particularidades, circunstancias, contexto, cronología y sociología del culto a Marte ${ }^{12}$ en el alto Bierzo. Por todo ello, este nuevo epígrafe dedicado a Mars Propitius solo nos permite constatar un nuevo e interesante epíteto clásico de la divinidad e incidir, de nuevo ${ }^{13}$, sobre la escasez de testimonios relativos al culto a Marte asociado a epítetos locales en el territorio de los astures, y, en general, en todo el Noroeste.

\section{BibliografíA}

Alarçao, J., Étienne, R. (dirs.), 1976, Fouilles de Conimbriga. II. Epigraphie et sculpture, Paris: Diffusion de Boccard.

Brouquier-Reddé, V., Bertrand, E. et alii (eds.), 2006, Mars en occidente. Actes du Colloque International Autour d'Allonnes (Sarthe), Les sanctuaires de Mars en Occident, Rennes: PUR. Archéologie et Culture.

Di Stefano Manzella, I., 1987, Mestiere di epigrafista. Guida alla schedatura del materiale epigrafico lapideo, Roma: Quasar.

Ernout, A., Meillet, A., 1932 [1994], Dictionnaire Étymologique de la Langue Latine. Histoire des mots, Paris : Éditions Klincksieck.

González Rodríguez, M. ${ }^{a}$ C., 2012, "Un ejemplo de romanización a través de la epigrafía latina altoimperial: los cultos indígenas en el Norte hispano», en: J. Santos, G. Cruz (eds.), Romanización, fronteras y etnias en la Roma antigua: el caso hispano, Vitoria-Gasteiz, 601-630 (Anejos de Veleia. Acta 12).

—,2013, "Revisones epigráficas del corpus de Gallaecia: nuevos hallazgos y viejos problemas», en: A. Hofeneder, P. De Bernardo Stempel (hgg), Théonymie celtique, cultes, interpretatio / Keltische Theonymie, Kulte, interpretatio. X. Workshop F.E.R.C.AN., Paris, 24-26 Mai 2010, [MPK 79], Wien, 11-25

Le Roux, P., 2006, "Mars dans la Péninsule Ibérique au Haut-Empire romain», en: V. Brouquier-Reddé, E. Bertrand et alii (eds.), Mars en occidente. Actes du Colloque International Autour d'Allonnes (Sarthe), Les sanctuaires de Mars en Occident, Rennes: PUR. Archéologie et Culture, 87-95.

Mangas, J., 1996, "Cultos minorasiáticos en el Noroeste de la Hispania romana», en: M. Á. Querol, T. Chapa (coords.), Homenaje al Profesor Manuel Fernández-Miranda, [Complutum Extra 6.1], Madrid: Universidad Complutense, 483-490.

Mañanes, T., 1982, Epigrafía y numismática de Astorga romana y su entorno, Salamanca: Museo de los Caminos de Astorga-Ediciones Universidad de Salamanca.

Scheid, J., 1990, Romulus et ses frères. Le collège des frères arvales, modèle du culte public dans la Rome des empereurs, [Bibliothèque des écoles françaises d'Athènes et de Rome, 275], Roma: École française de Rome.

Tranoy, A., 1981, La Galice romaine: recherches sur le nord-ouest de la péninsule ibérique dans l'Antiquité, Paris: Diffusion de Boccard.

\section{Abreviaturas}

AE L'Année Épigraphique, Paris: Universitè de Paris I, CNRS.

CIL HüBner, A., 1869- , Corpus Inscriptionum Latinarum, Berolini: G. Reiner.

12 Sobre los poderes y funciones del dios romano Marte en el occidente del imperio vid. las contribuciones recogidas en Brouquier-Reddé, Bertrand et alii (eds.), 2006.

13 González Rodríguez 2012 y 2013, 16-17. Incluso el epíteto Tillenus, considerado habitualmente como local, podría ser de importación minorasiática, tal y como ha apuntado Mangas 1996, 487. Si esta hipótesis es correcta entre los astures no habría ningún epíteto local asociado a Marte y en este territorio solo contaríamos, por el momento, con epítetos mediterráneos. 
EDCS Epigraphische Datenbank Clauss Slaby, www.manfredclauss.de

ERPLe Rabanal Alonso, M. A., S. M. a García Martínez, 2001, Epigrafía romana de la provincia de León: revisión y actualización, León: Universidad de León.

IRCP EnCARnaÇAO, J. d', 1984, Inscriçoes romanas do conventus Pacensis: subsidios para o estudo da romanizaçao, Coimbra: Instituto de Arqueologia da Faculdade de Letras.

RIB Collingwood, R. and Wright, R. P. (dir.), 1965, The Roman Inscriptions of Britain. I, Oxford: Oxford University Press.

RIT Alföldy, G., 1975, Die römischen Inschriften von Tarraco, Berlin: Walter de Gruyter \& co.

TitAq Kovács, P., Szabó, Á., 2009, Tituli Aquincenses I, Budapest: Pytheas. 